\title{
A new technology of GPS for field test drivers
}

\author{
Xiaojie $\mathrm{Jia}^{1, \mathrm{a}}$, Jie Kang ${ }^{1, \mathrm{~b}}$ and Yan Zhao ${ }^{2, \mathrm{c}}$ \\ ${ }^{1}$ Shijiazhuang HuaYan transportation science and technology limited company, \\ Shijiazhuang, 050081, China \\ ${ }^{2}$ Hebei Province People's Hospital, Shijiazhuang, 050057, China \\ a2511705376@qq.com, bkang.jie@126.com, 2385215931@qq.com
}

Keywords: high precision; GPS system; field test

Abstract. This paper introduces a kind of field test system of appraisal driver application in GPS technology inspection. Through two sets of GPS devices installed on the driver test vehicle and difference base station established on the ground, the contour coordinates of outside the vehicle movement state are get, comparing with specific test area information to determine whether a test vehicle pressing line, and get the discretion the vehicle pass the exam or not.

\section{Introduction}

With the development of the society and the rising of people's living standard, automobile has become a kind of fashion of people's life. The number of private cars increases sharply and raises a hot wave of varying degree student driver driving around the country. Following is the problem related to the transportation system of safe driving.

Statistics show that in the road traffic accident recent years in our country the motor vehicle driver factors accounted for $78.6 \%$, vehicle mechanical failure for $3.8 \%$ and the road condition factors for $0.17 \%$. Visible, the driver plays a decisive role in the traffic safety. Driver is one of the important link is that he should not only get information about roads and the vehicle running status information to process the information and take corresponding action to control vehicles. So must take good examination pass for drivers. In security training examination strictly accord with the requirements of the ministry of public security.

The traditional examination system mostly adopts magnetic, optical and electric combination to design implementation. Along with the continuous renewal of test standards and requirements, the current examination system no matter from the reliability, stability, having some shortcomings and has not completely applicable to test stable and reliable. Coupled with early on examination room hardening surface and pipe laying, later go line slot, the magnet embedding, all kinds of photoelectric pillar construction, all those require a lot of investment and not only waste of manpower also increasing the duration of the project. Therefore, the motor vehicle drivers test urgently needs a kind of examination system not affected by any weather, and speed, time saving, high efficiency, multifunctional and mobile positioning.

\section{Development of the system}

Working principle. Before the exam, according to the evaluation criteria of the exam project, project is divided into the corresponding evaluation area on a benchmark of the project test in site to the assessment. Then using a hand-held tool of surveying to survey the edge of each assessment project evaluation area, and using this hand-held tool to survey the contour of the test vehicle and the location of the tire relative to the center of the vehicle, and input the measurement result to the on-board computer in advance forming the map files, as shown in Figure 1. By two GPS devices mounted on vehicles in the exam get real-time information of vehicle location and through the calculation of the relative position of two GPS devices obtain the direction of the vehicle, and the outline of the vehicle and tire point position. According to the body and wheels in the judging area exam real-time whether examinee qualified or corresponding points penalty. 
Specific implementation. The examination control center is built in the examination room and the venue construction internal test server processing equipment including installation, machine, terminal management, wired and wireless LAN, wireless local area network (LAN) using WIFI way and the on-board computer communication.

Area in the center of the examination site to install the GPS base station equipment and digital radio through communication cables, GPS base station equipment and GPS satellite signal difference signals to digital radio, digital radio broadcast in the form of radio waves into the venue.

Two GPS devices A and B with $2 \mathrm{~mm}$ positioning accuracy installed at the top of the test vehicle along the centerline, the distance is not less than $1 \mathrm{~m}$ between the two GPS devices, as shown in figure 1. There is vehicle station equipment in test vehicle and connect with GPS devices A and B by communication cable connection. Vehicle station will receive the differential signal transmission and transfers to GPS devices A and B. GPS devices A and B get the accurate positioning information integrated itself via satellite positioning information combined with base station difference information and passing to an on-board computer. On-board computer is responsible for the GPS information collection and processing, calculation, the edge location of the judgment of the vehicle location information, and instructs the next operation of the motor vehicle drivers, and is responsible to upload evaluation results to the control center.

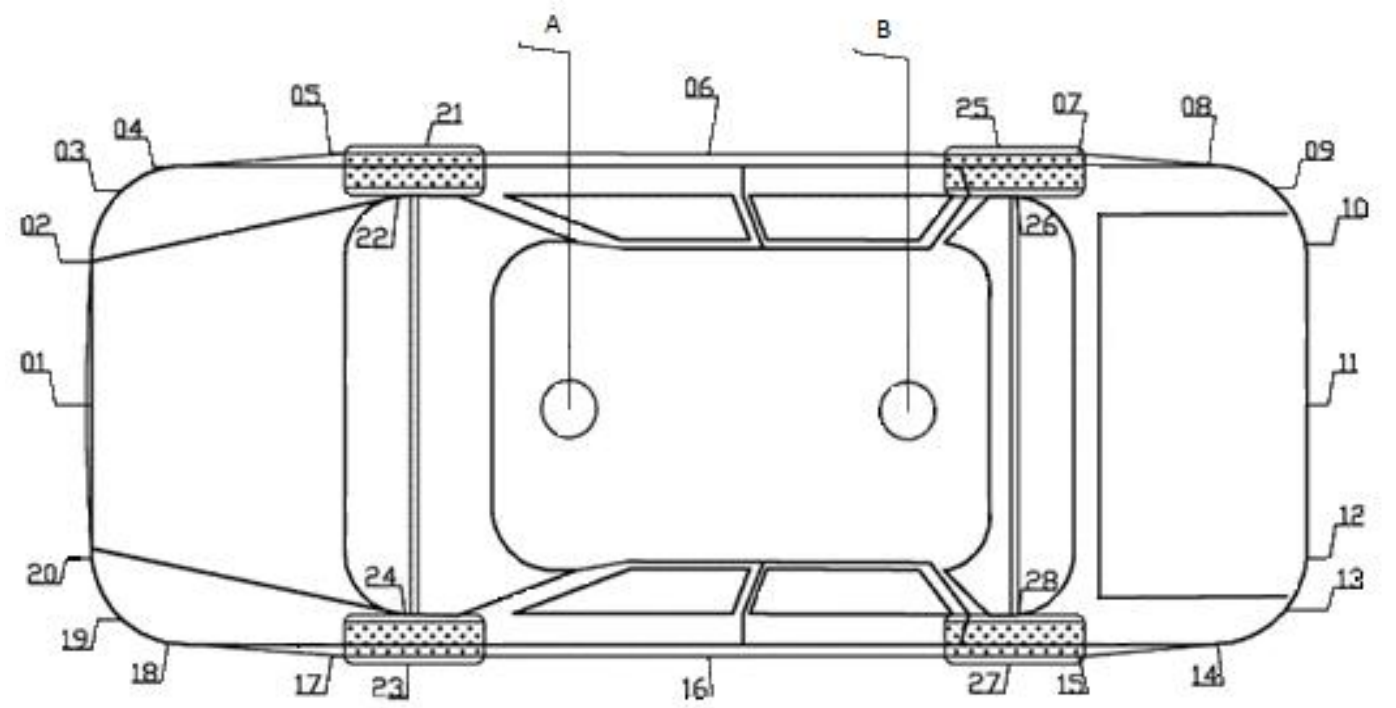

Fig.1. The sketch for test vehicle silhouette

According to the evaluation criteria of the project to divide test site into the corresponding evaluation area basing on the test project as benchmark. Using the positioning accuracy $2 \mathrm{~mm}$ GPS equipment to survey the edge of judging area of each test item in accordance with the last step and to test vehicle outline and the center of the vehicle tire position relative to vehicle.

Test-taking process: in the exam by GPS devices installed in vehicles to get the real-time information about the vehicle location and requiring acquisition frequency being not less than 4 times per second, through calculation of the relative position of two GPS information to obtain the direction of the vehicle and the position of each point of the vehicle silhouette and tire point. According to the judging area of the body and wheels to determine whether a test vehicle out of marker or rolling pressure and draw a conclusion that whether the examinee examination qualified or get corresponding points.

Below with "side parking" test project as an example to explain the process of examination judgment and side parking judging area diagram is shown in figure 2.

The vehicle body entering into 1 area, voice prompt "please enter side parking project";

The vehicle body entering into 2 areas, voice prompt "has entered the side parking";

The vehicle body entering into 8 areas, voice prompt "don't drive accordance with the prescribed route"; 
Every time the wheel into 3 zones, 10 points;

when selecting reverse found, the voice prompt "reverse";

The vehicle body entering into 4 area, the voice prompt "began to put in storage;

Every time the wheel into 6 zones, 10 points;

The vehicle body entering into 1 area, judge not according to stipulations route, unqualified;

When the vehicle is not reverse, do the following:

Vehicle body in 2 parts and out of the DA line, not qualified;

Vehicle body in 5 parts and out of the AB line, not qualified;

Vehicle body in 6 parts and out of the BC line, not qualified;

Vehicle body in 7 parts and out of the CD line, not qualified;

If the body only in 4 areas, qualified.

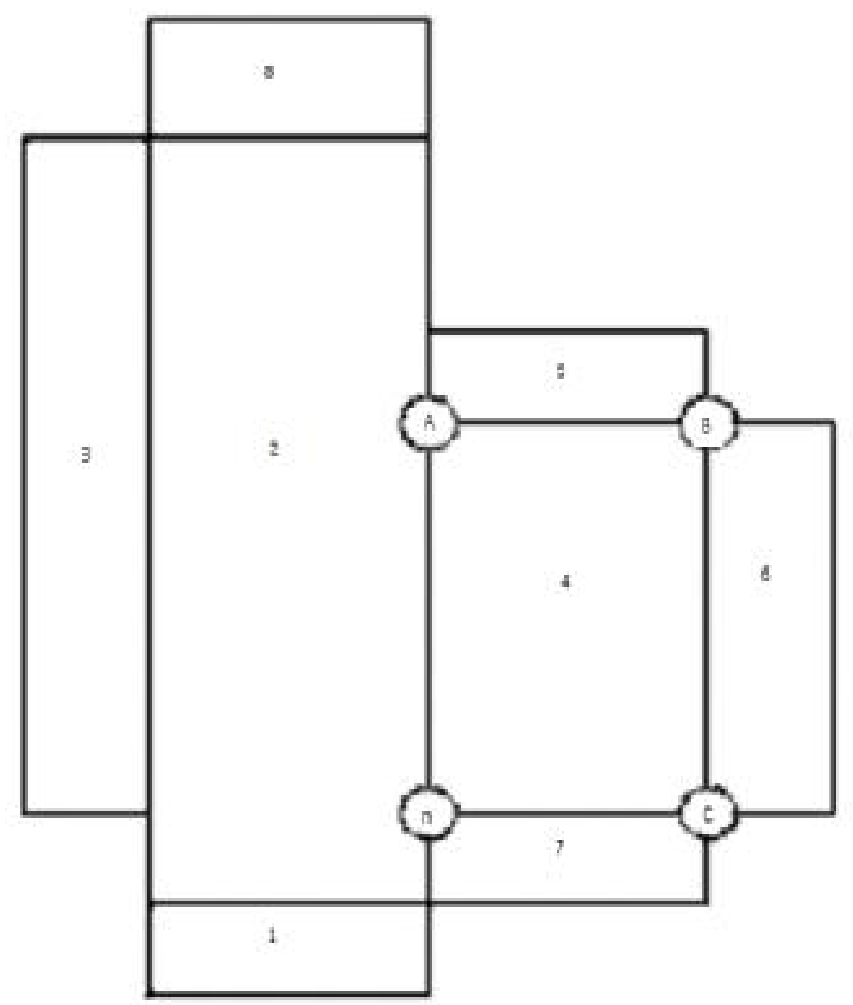

Fig. 2. The schematic of area test items for side parking

Electrical system. Electrical systems consist of control system, computer software etc. The control system consist of industrial on-board computer, IO adapter, signal conditioning circuit, infrared electric switch etc. completing detection and acquisition etc. for signal. Computer software system adopts the popular Borland c++ Builder and using Microsoft SQL 2000 for data management system, application connecting with the database. All these provides a powerful guarantee to improve the efficiency of programming and the reliability of the data management.

\section{Conclusion}

After repeating comparison tests, confirmed that the use of the system of field testis high accuracy, appraisal and accurately and simple installation, convenient operation and use.

Because the GPS device adopts RTK (Real time kinematic) the real-time dynamic difference technology in the system and the positioning accuracy is $2 \mathrm{~mm}$, even if maximum error of the vehicle front is less than $1 \mathrm{~mm}$, it yet meet the require of national standard for test equipment. After using high precision GPS positioning system, the system greatly improves the reliability and application of the examination system. GPS is clock and not influenced by any weather, global coverage $(98 \%)$, three 
dimensional time and speed with high precision, fast, time saving, high efficiency, wide application, multi-function, mobile positioning.

Especially with the latest test standard requirements has increased a lot the new test program, and some projects have been unable to use ordinary photoelectric signal to evaluate, and using the GPS devices can easily solve these problems also simplified a lot of original test program. Even the standard upgrade again, increasing or decreasing the test project, or adjusting the examination standard, the system only requires adjusting the map information software in the system and don't need to add or modify any hardware equipment, then greatly reduce the upgrade cost and time, and achieve the result once and for all.

\section{References}

[1] GA/T 1026-2012 Driving test contents and methods.

[2] GA/T 1028.3-2012 General technical specifications for driving test systems-Part 3: Field driving test systems.

[3] GA/T 1029-2012 Layout specifications for driving fields and facilities.

[4] GA/T 1030.1-2012 Acceptance specifications for driving test fields and driving test systems-Part 1: Test fields.

[5] GA/T 1030.2-2012 Acceptance specifications for driving test fields and driving test systems-Part 2: Test systems. 